Ann. Biol. anim. Bioch. Biophys., I974, 14 (4-B), 8I3-8I9.

\title{
INFLUENCE DE QUELQUES FACTEURS ALIMENTAIRES SUR L'AMINOACIDÉMIE LIBRE DE VACHES LAITIÈRES REGEVANT DES RATIONS A BASE D'ENSILAGE DE MAÏS
}

\author{
C. CHAMPREDON, R. PION et R. VÉRITE** \\ Laboratoire d'Étude du Métabolisme azoté, \\ * Laboratoire de la Production laitière, \\ Centre de Recherches de Clermont-Ferrand, I. N.R.A., \\ Theix, 63110 Beaumont
}

\section{RÉSUMÉ}

Les concentrations sanguines en acides aminés libres, en glucose et en urée ont été déterminées au cours d'une expérience factorielle $2 \times 2 \times 2$ réalisée avec 24 vaches laitières. Les régimes expérimentaux permettaient de comparer 2 ensilages de maïs (normal ou enrichi en grains), deux taux énergétiques, et deux compléments azotés (arachide ou arachide-urée).

Les concentrations en acides aminés indispensables et semi-indispensables du sang étaient plus faibles dans les échantillons obtenus au cours de la période expérimentale que dans ceux qui ont été prélevés à la fin des périodes préexpérimentale ou post-expérimentale. L'ensilage enrichi en grains a permis une meilleure production laitière au prix d'une diminution de teneurs en certains acides aminés indispensables đu sang. L'élévation du taux énergétique du régime a également permis d'améliorer la production laitière mais sans modification notable de l'aminoacidémie, alors que le remplacement d'une partie de l'arachide par de l'urée n'a eu d'influence que sur la teneur en histidine libre du sang. La glycémie était abaissée par la distribution de l'ensilage enrichi en grains alors que l'urémie était augmentée à la suite de l'ingestion de ce même régime ainsi que par la présence d'urée dans la ration.

Les médiocres performances laitières obtenues surtout avec l'ensilage de maîs laiteux, résultent de carences en acides aminés (méthionine, lysine et histidine) dues principalement à des synthèses insuffisantes dans le rumen.

I'influence sur la production laitière de l'ingestion de rations à base d'un ensilage de maïs laiteux, ou d'un ensilage réalisé à partir đu même maïs enrichi en grains, a été étudiée sur des vaches en milieu de lactation (VÉRITÉ et JouRNET, I975 a). Nous avons cherché atı cours de cette expérience à déterminer 1'influence de la nature du régime sur les teneurs du sang des animaux en acides aminés libres (PION, I972), en urée et en glucose, afin de relier les performances des animaux au taux de satisfaction des besoins en acides aminés. 
Les conditions expérimentales et les régimes ont été décrits en détail par ailleurs (VÉRITÉ et JOURNET, I975 a). Au cours de la période pré-expérimentale (3 semaines), les vaches recevaient un régime commun (foin de graminées + ensilage d'herbe + betteraves + concentré) selon leurs besoins. Pendant la période expérimentale (ro semaines), les vaches ont été réparties en 8 lots de 3 selon un schéma factoriel permettant d'apprécier l'influence de la nature de l'ensilage de mais (laiteux ou enrichi en grains), du niveau des apports énergétiques (Ioo ou IIo p. Ioo des besoins) et de la nature du complément azoté (tourteau d'arachide seul ou avec urée). Au cours de la période post-expérimentale (3 semaines) toutes les vaches ont reçu à nouveau un régime commun (foin de graminées + pulpes sèches de betteraves + ensilage de maïs + concentré) selon leurs besoins.

Les différents régimes expérimentaux n'ont permis qu'une médiocre persistance de la production laitière; l'ingestion de l'ensilage enrichi en grains a conduit à une production laitière plus élevée, et à un moindre engraissement des animaux que celle de l'autre ensilage. L'élévation du taux énergétique du régime a entraîné une augmentation de la production laitière, tandis que la nature du supplément azoté ne semblait pas avoir d'influence sur les performances des animaux.

Des prélèvements de sang jugulaire étaient effectués à la fin de la préexpérience, au cours des deuxième, quatrième et dixième semaines de l'expérience et à la fin de la post-expérience. Les échantillons nécessaires au dosage des acides aminés libres étaient constitués en regroupant des quantités égales du sang des 3 animaux de chaque lot, sauf dans le cas de la période préexpérimentale où les sangs des 24 vaches étaient mélangés au moment du prélèvement.

I,es échantillons de sang destinés à la détermination de l'aminoacidémie libre étaient mélangés immédiatement après le prélèvement à 7 fois environ leur volume d'éthanol $95^{\circ}$ froid contenant 2 p. Ioo de thiodiglycol. Les acides aminés libres étaient extraits avec de l'éthanol $82^{\circ}$ par épuisements successifs, et dosés par chromatographie sur résine échangeuse d'ions (SPACkman, StEIn et MoORE, I958). Les échantillons de sang destinés au dosage du glucose et de l'urée plasmatiques étaient placés dès le prélèvement dans des tubes héparinés partiellement immergés dans de la glace fondante. I,es dosages de glucose et d'urée étaient effectués à partir du plasma correspondant à chaque animal respectivement par les méthodes colorimétriques à la glucose oxydase et à la diacétyl monoxime (Mrché, I97 I).

\section{RÉSULTATS}

L'évolution moyenne dans le temps de l'aminoacidémie libre des 24 vaches et l'influence des trois facteurs nutritionnels étudiés sur l'aminoacidémie libre sont rapportées dans le tableau $\mathrm{I}$.

Les concentrations en acides aminés indispensables et en tyrosine (tabl. I) sont notablement plus faibles dans les échantillons prélevés au cours des $4^{\mathrm{e}}$ et Io $^{\mathrm{e}}$ semaines de la période expérimentale qu'à la fin de la préexpérience. Elles redeviennent généralement voisines de celles qui étaient observées au cours de la préexpérience dans les échantillons prélevés à la fin de la période post-expérimentale. I,es diminutions de teneurs sont particulièrement nettes pour les acides aminés à chaîne 


\section{TABLEAU I}

Aminoacidémie libre des vaches laitièves ( $\mathrm{mg} \mathrm{p}$. Ioo $\mathrm{g}$ de sang frais)

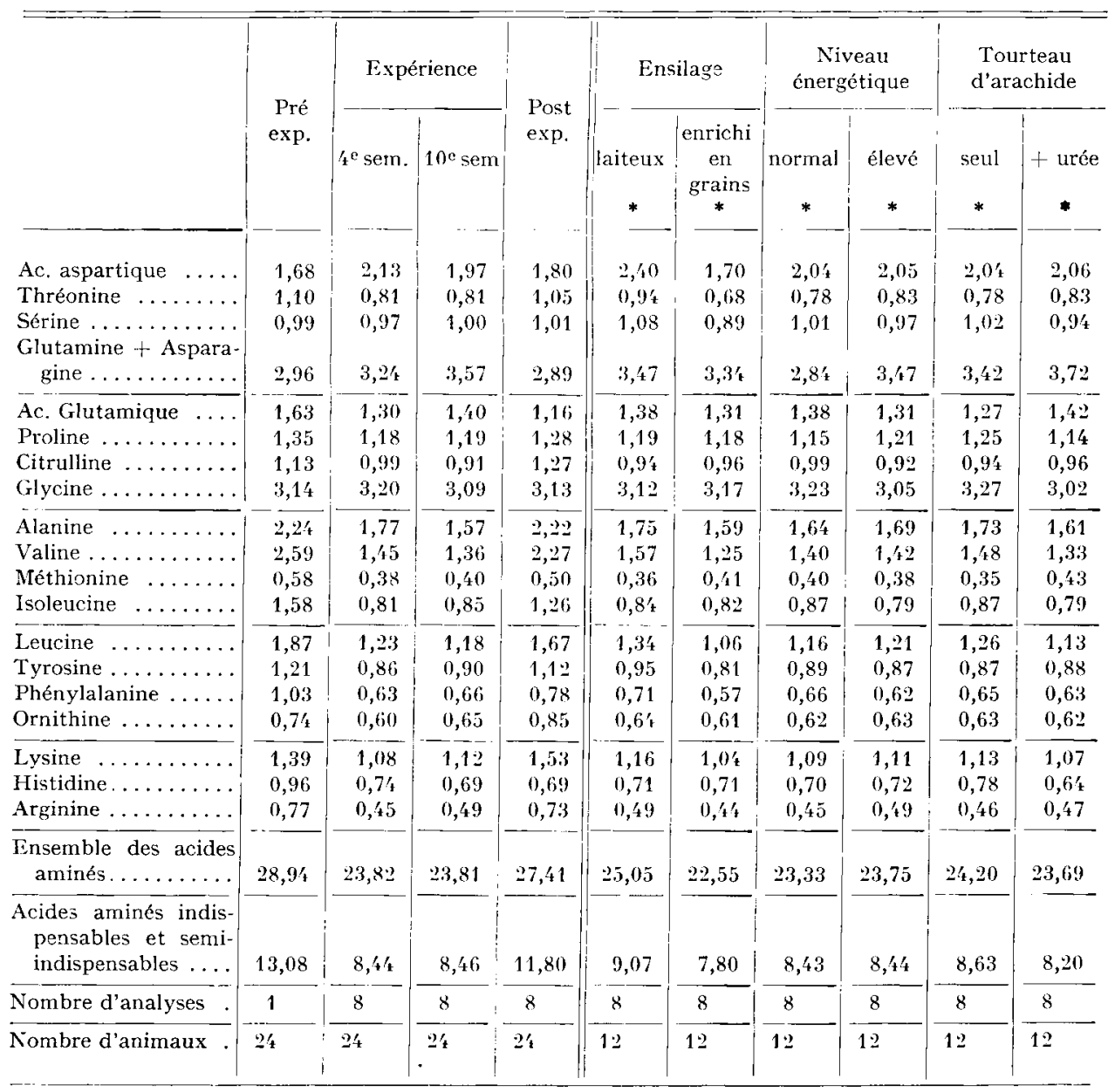

* : moyenne des valeurs observées en $4^{\mathrm{e}}$ et $10^{\mathrm{e}}$ semaines de la période expérimentale.

ramifiée (leucine, isoleucine, valine) et la phénylalanine, dont les concentrations restent d'ailleurs inférieures à leur niveau initial jusqu'à la fin de la période postexpérimentale. Les teneurs en acides aminés non indispensables sont généralement peu modifiées par les changements de régime alimentaire; le fait le plus marquant est la diminution de teneur en alanine associée à une augmentation de teneur en glutamine (plus asparagine) apparaissant au cours de la période expérimentale. L'urémie (tabl. 2) augmente de la deuxième à la quatrième semaine d'expérience, puis diminue ensuite; les teneurs en glucose plasmatique sont plus élevées en période post-expérimentale que durant l'expérience.

La distribution de l'ensilage enrichi en grains at lieu de l'ensilage laiteux se 
traduit par un abaissement notable des teneurs en certains acides aminés indispensables et semi-indispensables, en particulier en thréonine, et à un moindre degré en valine, leucine, phénylalanine et tyrosine. Il en est de même pour deux acides aminés non indispensables, la sérine et l'acide aspartique. En revanche, les teneurs en méthionine, isoleucine, lysine et histidine ne sont pas modifiées. La glycémie est diminuée, alors que l'urémie est augmentée.

\section{TABLEAU 2}

Teneurs en urée et en glucose du plasma des vaches laitières

(mg p. Ioo $\mathrm{ml}$ de plasma)

\begin{tabular}{|c|c|c|c|c|c|}
\hline & & \multicolumn{3}{|c|}{ Période expérimentale } & \multirow{2}{*}{$\begin{array}{l}\text { Période post- } \\
\text { expérimentale }\end{array}$} \\
\hline \multicolumn{2}{|c|}{ Semaine de prélèvement } & $2 \mathrm{e}$ & $4^{\mathrm{e}}$ & $10^{\mathrm{e}}$ & \\
\hline Ensilage laiteux & $\begin{array}{l}\text { Urée } \\
\text { Glucose }\end{array}$ & $\begin{array}{l}25,9 \\
61,6\end{array}$ & $\begin{array}{l}33,0 \\
63,6\end{array}$ & $\begin{array}{l}23,1 \\
64,3\end{array}$ & $\begin{array}{l}27,2 \\
67,7\end{array}$ \\
\hline $\begin{array}{l}\text { Ensilage enrichi } \\
\text { en grains }\end{array}$ & $\begin{array}{l}\text { Urée } \\
\text { Glucose }\end{array}$ & $\begin{array}{l}29,5 \\
57,5\end{array}$ & $\begin{array}{l}39,7 \\
55,0\end{array}$ & $\begin{array}{l}24,7 \\
60,7\end{array}$ & $\begin{array}{l}26,0 \\
65,9\end{array}$ \\
\hline $\begin{array}{c}\text { Niveau énergétique } \\
\text { normal }\end{array}$ & $\begin{array}{l}\text { Urée } \\
\text { Glucose }\end{array}$ & $\begin{array}{l}27,7 \\
60,4\end{array}$ & $\begin{array}{l}37,7 \\
58,0\end{array}$ & $\begin{array}{l}24,6 \\
64,4\end{array}$ & $\begin{array}{l}26,4 \\
65,3\end{array}$ \\
\hline $\begin{array}{l}\text { Niveau énergétique } \\
\text { élevé }\end{array}$ & $\begin{array}{l}\text { Urée } \\
\text { Glucose }\end{array}$ & $\begin{array}{l}27,8 \\
58,5\end{array}$ & $\begin{array}{l}35,4 \\
60,1\end{array}$ & $\begin{array}{l}23,2 \\
60,7\end{array}$ & $\begin{array}{l}26,7 \\
68,0\end{array}$ \\
\hline $\begin{array}{c}\text { Tourteau d'arachide } \\
\text { seul }\end{array}$ & $\begin{array}{l}\text { Urée } \\
\text { Glucose }\end{array}$ & $\begin{array}{l}25,6 \\
57,3\end{array}$ & $\begin{array}{l}33,4 \\
58,0\end{array}$ & $\begin{array}{l}23,9 \\
59,8\end{array}$ & $\begin{array}{l}26,9 \\
65,3\end{array}$ \\
\hline $\begin{array}{c}\text { Tourteau d'arachide } \\
+ \text { urée }\end{array}$ & $\begin{array}{l}\text { Urée } \\
\text { Glucose }\end{array}$ & $\begin{array}{l}30,1 \\
61,8\end{array}$ & $\begin{array}{l}39,8 \\
60,3\end{array}$ & $\begin{array}{l}23,9 \\
66,9\end{array}$ & $\begin{array}{l}26,2 \\
68,8\end{array}$ \\
\hline
\end{tabular}

L'augmentation du taux énergétique de la ration ne modifie pas l'aminoacidémie libre des animaux, mais entraine une nette augmentation de la teneur en glutamine (plus asparagine) sans modification notable de l'urémie.

La substitution partielle d'urée au tourteau d'arachide n'entraîne que des modifications de faible amplitude, sauf en ce qui concerne 1'histidine dont la concentration sanguine est diminuée, et l'uré plasmatique dont la teneur est nettement augmentée sauf à la fin de l'expérience.

\section{DISCUSSION}

Bien que les vaches aient été alimentées normalement ou avec un léger excès d'azote et d'énergie durant la période expérimentale, il semble que les rations utilisées n'aient pas permis de mettre à la disposition de la glande mammaire des 
quantités suffisantes d'acides aminés indispensables pour synthétiser les matières azotées du lait. Ce phénomène pourrait être lié à la forte solubilité de l'azote alimentaire ou à un apport de composants énergétiques qui serait soit insuffisant, soit inadéquat pour une bonne utilisation des matières azotées alimentaires dans le rumen.

Avec 1'ensilage enrichi en grains, les diminutions importantes des teneurs sanguines de la plupart des acides aminés indispensables et semi-indispensables peuvent s'expliquer par le fait que les vaches ont produit davantage de lait tout en ingérant une plus faible quantité de matières azotées, et traduire une meilleure utilisation de l'azote du régime. De même, la diminution de la glycémie reflète une utilisation élevée du glucose par la glande mammaire pour la synthèse de lactose du lait.

Cette amélioration de l'utilisation de l'azote alimentaire peut sans doute s'expliquer par un meilleur équilibre du mélange d'acides aminés fournis à l'organisme par le tube digestif. En effet, les teneurs en méthionine, lysine et histidine libres du sang qui étaient très faibles, ne sont que peu ou pas diminuées et augmentent en valeur relative malgré la demande élevée d'acides aminés par la glande mammaire, ce qui avait déjà été observé (REMOND et al., I97I) chez des vaches laitières recevant un régime supplémenté en DL-méthionine. Ces trois acides aminés seraient limitants pour la production laitière (ChAMpredon, I972) dans le cas de l'ensilage laiteux, car ils seraient présents en quantités plus faibles dans les contenus digestifs qu'avec l'ensilage enrichi en grains.

Ce phénomène peut être la conséquence de la teneur élevée des grains de maïs en acides aminés soufrés, qui a eu pour effet un apport accru de ces composés dans l'intestin, associée à une augmentation du nombre de protozoaires du rumen dont les matières azotées sont notablement plus riches en lysine et surtout en histidine que celles des bactéries (FAuconneau et Pion, I966).

L'augmentation de l'urémie observée chez les animaux recevant l'ensilage enrichi en grains peut provenir du fait que l'ammoniogenèse dans le rumen est plus importante avec cet aliment qu'avec l'ensilage laiteux (Vf́rITÉ et JoURNET I975 $b$ ), ou être la conséquence de l'utilisation à des fins énergétiques de certains acides aminés et en particulier des acides aminés non indispensables (acide aspartique, alanine, sérine...).

Le fait que l'aminoacidémie libre des vaches laitières ne soit pratiquement pas diminuée par l'élévation du taux énergétique de la ration montre que la synthèse protéique est vraisemblablement limitée par l'apport quantitatif d'énergie dans le rumen. En effet, 1'augmentation de la quantité d'énergie distribuée aux animaux a eu pour conséquence l'augmentation de la quantité de matières azotées excrétées dans le lait. Comme l'aminoacidémie libre des vaches est peu modifiée, il est vraisemblable qu'une fourniture accrue d'acides aminés à partir du tube digestif aura permis de compenser totalement le supplément de besoin consécutif à l'élévation de la quantité de lait produite. On observe aussi une teneur plus élevée en amides (glutamine + asparagine) lorsque les animaux recevaient le régime le plus riche en énergie. Ce résultat pourrait provenir d'un transport accru de l'ammoniac du rumen sous forme de glutamine synthétisée à partir de chaines hydrocarbonées provenant de la transformation de nutriments énergétiques.

Le remplacement partiel du tourteau d'arachide par de l'urée dans les rations, ne provoque au niveau sanguin qu'une augmentation de l'urémie et une diminution de la teneur en histidine; les faibles différences observées peuvent s'expliquer par le 
fait que l'urée apportée ne représente qu'un faible pourcentage (I6 p. Ioo) de l'azote total de la ration et qu'une part importante des matières azotées des différents régimes ingérés est fortement dégradable dans le rumen.

Ces résultats sont en accord avec ceux obtenus précédemment avec des vaches laitières recevant de l'ensilage de maîs et un apport complémentaire d'azote sous forme d'urée ou de tourteau d'arachide (Champredon, Pion, Journe't i970). Des augmentations ou diminutions de teneurs sont observées pour les mêmes acides aminés dans les 2 expériences, sauf dans le cas de l'acide glutamique et de la glycine ; l'amplitude des écarts observée au cours de la présente expérience est beaucoup plus faible que celle qui avait été mise en évidence précédemment, alors que l'azote uréique ingéré atteignait 29 p. Ioo de l'azote total.

\section{CONCLUSION}

La médiocrité de la production laitière obtenue avec les rations expérimentales et en particulier avec l'ensilage de maïs laiteux semble provenir de carences en certains acides aminés indispensables (méthionine, lysine, histidine) dues au moins en partie à des synthèses insuffisantes dans le rumen. L'addition de grains de maïs à l'ensilage ou une augmentation du taux énergétique des rations entraîne une amélioration de l'utilisation des matières azotées des régimes.

Reçu pour publication en juin 1974.

\section{REMERCIEMENTS}

Nous remercions M. M. C. MicheL qui a bien voulu effectuer les dosages de glucose et d'urée plasmatiques.

\section{SUMMARY}

EFFECT OF SOME DIETARY FACTORS ON LEVELS OF BLOOD FREE AMINO ACIDS IN DAIRY COWS FED MAIZE SILAGE RATIONS

The effect of nutrition on the concentration of free amino acids, urea and glucose in the blood was studied in a $2 \times 2 \times 2$ factorial experiment with 24 lactating cows. The experimental treatments consisted of maize silage with or without grain enrichment, two levels of energy intake (IOO and IIO p. IOO of estimated requirements) and a nitrogen supplement of peanut meal or peanut meal plus urea (VÉRITÉ et JouRNET, I974a).

Essential amino acids and tyrosine in the blood (table $I$ ) were lower during the experimental period than before and after, when all the cows were fed the same diets containing hay. Grain enriched silage resulted in an increase of milk production and a decrease in free essential amino acids in the blood. Increasing the energy content of the diet had no effect on the blood amino acid patterns but increased milk production. The substitution of part of the peanut meal with urea decreased the level of free histidine in the blood. Plasma glucose was lower and plasma urea higher (table 2) for the grain enriched silage than with the control silage; plasma urea level was increased by feeding urea to the cows. 
From these results it may be inferred that the rather poor milking performances of the animals are related to amino acid deficiencies (methionine, lysine and histidine) due to inadequate protein synthesis in the rumen.

\section{RÉFÉRENCES BIBLIOGRAPIIIQUES}

Champredon C., 1972 . Utilisation de l'aminoacidémie libre comme mesure de la satisfaction du besoin en certains acides aminés des chèvres laitières. Étude de quelques facteurs. Thèse de Doctorat de spécialité (Physiologie), Clermont-Ferrand.

Champredon C., Pion R., Journet M., i97o. Influence de la nature des matières azotées alimentaires sur l'aminoacidémie libre des vaches laitières. Ann. Biol. anim. Bioch. Biophys., 10, 5I7-52I.

Fauconneau G., Pion R., 1966. Amino acid composition of proteins of some fodder plants. Proc. IX Internat. Grassland Congr., 779-781.

Micher M. C., 1971. Analyse quantitative de quelques substances azotées et glucidiques en milieu biologique. Thèse Doctorat d'Université, Clermont Ferrand.

Pion R., I972. In de Cuenca C. L., VAnbelle M. Protéines et acides aminés en nutrition humaine et animale, 529-542. G.A.R.S.I., Londres, Madrid.

Remond B., Champredon C., Decaen C., Pion R., Journet M., i97i. Influence d'un apport de DL-Méthionine à des vaches au début de la lactation sur la production laitière et la composition du sang. Ann. Biol. anim. Bioch. Biophys., 11, 455-469.

Spackman D. H., Stein W. H., Moore J., I958. Automatic recording apparatus for use in the chromatography of amino acids. Analyt. Chem., 30, I I90-ı 206.

Vérité R., Journet M., I975 $a$. Alimentation des vaches laitières avec de l'ensilage de mais: influence de la nature de l'ensilage de la suralimentation énergétique et de la nature de la complémentation azotée. I. Production laitière. Ann. Zootech. (sous presse).

Vérité R., Journet M., I975 $b$. Alimentation des vaches laitières avec de l'ensilage de mais: influence de la nature de l'ensilage, de la suralimentation énergétique et de la nature de la complémentation azotée. II. Digestion dans le rumen. Ann. Zootech. (sous presse). 